\title{
A Pipe Inspection Using a Circumferential SH-Mode Plate Wave Generated in a Pipe by an Electromagnetic Acoustic Transducer (EMAT)
}

\author{
Riichi Murayama1, Hideo Nishino² \\ ${ }^{1}$ Faculty of Engineering, Fukuoka Institute of Technology, Fukuoka, Japan \\ ${ }^{2}$ Graduate School of Advanced Technology and Science, Tokushima University, Tokushima, Japan \\ Email: murayama@fit.ac.jp
}

How to cite this paper: Murayama, R. and Nishino, H. (2018) A Pipe Inspection Using a Circumferential SH-Mode Plate Wave Generated in a Pipe by an Electromagnetic Acoustic Transducer (EMAT). World Journal of Engineering and Technology, 6, 671-683. https://doi.org/10.4236/wjet.2018.63043

Received: July 10, 2018

Accepted: August 19, 2018

Published: August 22, 2018

Copyright $\odot 2018$ by authors and Scientific Research Publishing Inc. This work is licensed under the Creative Commons Attribution International License (CC BY 4.0).

http://creativecommons.org/licenses/by/4.0/

\begin{abstract}
Inspection of a pipeline is essential for the safe use of such facilities. A trial sensor using an electromagnetic acoustic transducer (EMAT), which can generate the $\mathrm{SH}$-mode plate wave propagating in the circumferential direction, has been developed to realize this objective. It consists of a circulating electromagnetic induction coil around the pipe and many permanent magnets arranged on the surface of the pipe in the circumferential direction. It is postulated that the intensity of the SH-mode plate wave propagating in the circumferential direction is dependent on any defects in the circumferential direction. A resonance method was then utilized to obtain a stronger received signal. As a result, it was confirmed that the resonance status can be detected. The relationship between the signal intensity and the pipe thickness was then evaluated. It was confirmed that the wall thickness of about $20 \%$ can be detected under a static condition. Finally, a moving test has been executed by using an axially traveling device manufactured by trial. The test pipes with different sizes of drilled holes were prepared. The change in the received signal intensity according to different sizes of the drilled holes was successfully detected.
\end{abstract}

\section{Keywords}

SH-Mode Plate Wave, EMAT, Pipe, Nondestructive Inspection, Resonance Method

\section{Introduction}

The quality monitoring of any materials, any equipment, and any structures is becoming very important for achieving a safe and secure society. Among them, 
the non-destructive evaluation is an important method for detecting the presence or absence of defects inside any materials and structures. There are many sensing methods to realize a nondestructive evaluation, mainly using light waves, radiation, ultrasonic waves, electromagnetic waves, magnetism, etc. The measurement principle is based on the reaction due to the internal abnormality or the presence of the defect. The ultrasonic method is a powerful tool among them because it can safely and conveniently inspect the internal aspects of any materials. Among them, a method using guide waves has been widely studied recently [1] [2] [3]. Many of the thermal power plants, nuclear power plants, petrochemical plants, and energy pipelines are aging. They also have many serious problems, such as the progress of corrosion of their pipes. Several practical tests have already been done and any problems involving on-site applications have also been reported [4] [5] [6] [7] [8]. The detection of the bottom failure of the rail of a crossing rail utilizing the guided wave propagating in the railroad rail has also started to be developed [9]. In addition, as a common problem of the inspection method using the guided wave, it must be improved to reduce any noise signal and to increase the received signal amplitude. For example, the noise from any peripheral machines is usually very strong and effective counter measures are required. In addition, in order to use non-contact sensors, such as an electromagnetic acoustic transducer (EMAT), it is possible to improve the $\mathrm{S} / \mathrm{N}$ ratio by signal processing as a method of compensating for the low conversion efficiency. There is a technique for effectively improving the $\mathrm{S} / \mathrm{N}$ ratio by applying a signal processing technique such as pulse compression.

Among the proposed methods, a guided wave propagating in the circumferential direction of a pipe is being studied as a method of inspecting pipes. For example, experimental and analytical results have revealed the frequency dispersion characteristics of a circumferentially propagating guided wave or the differences and similarities of the characteristics compared to a flat plate [10]. On the practical side, corrosion tests using the circumferentially propagating guided wave were investigated [11] [12]. A pipe screening technology using the circumferentially propagating Shear Horizontal (SH) plate waves and axial traveling equipment was also evaluated [13].

In this research study, a sensor system that transmits and receives the $\mathrm{SH}$-mode plate wave propagating in the circumferential direction of a pipe has been developed. It uses an EMAT to mechanically move the ultrasonic sensor in the axis direction of a pipe without any contact medium. It was then discussed whether it is possible to detect the presence or absence of any defects in a pipe while mechanically running in the axis direction of a pipe. The basic configuration of a trial EMAT consists of an electromagnetic induction coil (EM-coil) wound in the circumferential direction and neodymium permanent magnets magnetized in the vertical direction installed at a constant interval in the circumferential direction. The drive frequency is adjusted to realize a resonance status according to the distance among the two magnets [14]. As for the trial EMAT, a meander line shape EM-coil finally wound in the circumferential di- 
rection of the pipe and the permanent magnet placed only on the axial direction part of the EM-coil was adopted. In this paper, an outline of the trial sensor system and the performance evaluation results under the static condition are described.

\section{Development of Synchronous Circumferential Propagating SH-Mode Plate Wave EMAT}

\subsection{Basic Concept of Inspection Method}

There are many guided wave modes propagating in the circumferential direction of a pipe, for example, the S-mode plate wave, A-mode plate wave, the SH-mode plate wave, etc. The SH-mode plate wave of zero order was finally chosen because it is considered not to be affected by the surface status of the pipe due to the oscillation direction characteristic. That is, as shown in Figure 1, it was attempted to detect any defects utilizing the signal intensity changes when the sensor is running over the defects in the pipe.

\subsection{Basic Structure of Synchronous Circumferential Propagating SH-Mode Plate Wave EMAT}

Figure 2(a) shows a sectional view of the trial EMAT. The SH-mode plate wave of the lowest order has a vibration orthogonal to the propagating direction in the plane. The vibrational Lorentz force in the axial direction was generated by interaction between the magnetic field and the high frequency electric current. The magnetic field is generated by a vertically-magnetized permanent magnet arranged in the circumferential direction on the pipe. The high frequency electric current is generated by the EM-coil wound in the circumferential direction [15]. As the Lorentz force periodically fluctuates according to the high frequency electric current, an SH-mode plate wave propagating in the circumferential direction of the pipe is also generated. Therefore, it is assumed that the received signal will be able to be dramatically amplified by selecting the drive frequency that synchronizes with the propagation time such that the SH-mode plate wave travels between two magnets. Figure $2(\mathrm{~b})$ is a developed view in which the magnets are arranged in the circumferential direction of the pipe. It shows that it functions as a synchronous type by adjusting the phase of the $\mathrm{SH}$-mode plate wave between the adjacent magnets.

\section{Basic Performance Evaluation Test}

For the basic performance evaluation test, a circular coil wound in the circumferential direction of the pipe was adopted for both the transmitter and receiver EM-coils. The driving device combines the waveform generator and the power amplifier to emit a burst-type sine wave into the transmitter EM-coil. In the receiving system, a preamplifier and a discriminator are combined to amplify by $80 \mathrm{~dB}$ and filter from $50 \mathrm{kHz}$ to $500 \mathrm{kHz}$. An analysis was performed by a personal computer using the signal waveform recorded by an oscilloscope. 

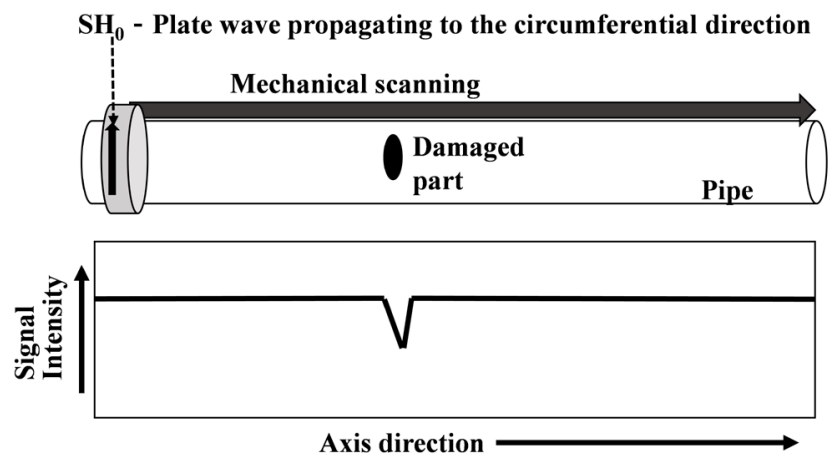

Figure 1. Fundamental concept of the developed ultrasonic inspection system.

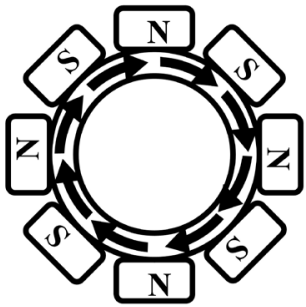

(a)

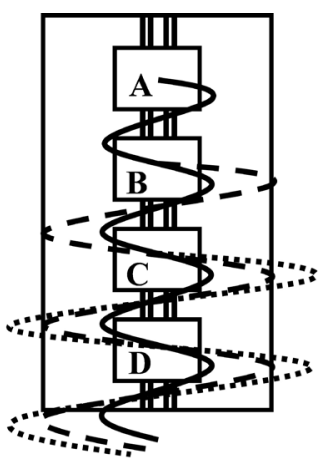

(b)

Figure 2. Basic structure of the trial EMAT-sensor for the $\mathrm{SH}$-mode plate wave traveling in the circumferential direction. (a) Sectional view; (b) Development.

\subsection{Experimental Conditions}

Some experiments for determining the sensor fabrication conditions were conducted using an aluminum pipe of $4000 \mathrm{~mm}$ length, $60 \mathrm{~mm}$ outer diameter, and $2 \mathrm{~mm}$ wall thickness as the test piece. The sensor was placed on the center of the pipe in the axis direction. In addition, the signal intensity was checked when changing the number of windings and the wire diameter of the EM coil. For the transmitter-EM coil, it was decided to use a copper wire with a wire diameter of $0.315 \mathrm{~mm}$, and 8 number of turns, and for the receiver-EM coil, a wire diameter of $0.315 \mathrm{~mm}$ and 20 number of turns. The distance between the transmitter and receiver-EM coil was $2 \mathrm{~mm}$. The number of cycles of the burst pulse was 40 .

\subsection{Optimization of the Drive Frequency}

The number of permanent magnets installed in the circumferential direction of the pipe was 8 . The relationship between the driving frequency and the signal strength was then investigated. The case fixing the permanent magnets was fabricated using polyethylene terephthalate (PET) as the material of choice. The size of the permanent magnet was $20 \times 22 \times 12 \mathrm{~mm}$ and the surface magnetic flux density was $405 \mathrm{mT}$. The expected resonant driving frequency was consi- 
dered to be when the half wavelength $(\lambda)$ of the $\mathrm{SH}_{0}$ mode plate wave coincided with the distance between the two magnets. The calculation result of the relationship between the number of cycles and the resonance frequency in the case of an aluminum pipe with a $60 \mathrm{~mm}$ outer diameter $\times 2 \mathrm{~mm}$ wall thickness was $68.1 \mathrm{kHz}$ [16]. As an example, the received signal waveform and its spectrum waveform at resonance $(71 \mathrm{kHz})$ and non-resonance $(50 \mathrm{kHz})$ is shown in Figure 3. It is noted that the resonance effect is sufficiently exhibited in the figure. In order to avoid the time domain affected by the induced electromagnetic waves due to the high-frequency electric current signal applied to the transmitter EM-coil, a Fourier analysis was performed for about $500 \mu$ s being (dead zone) affected by the induced electromagnetic wave. Figure 4 shows the relationship between the drive frequency and the intensity of the frequency spectrum. In addition, in order to obtain the minimum impedance in the case of the transmitter EM-coil and the maximum impedance in the case of the receiver EM-coil, a capacitor was added between the EM-coil and the electronic circuit according to formula (1). Some capacitors are connected in series with the transmitter EM-coil and in parallel with the receiver EM-coil. The experiments were conducted at a 1 $\mathrm{kHz}$ pitch. The spectrum intensity increased by about 10 times higher than the other drive frequencies. The frequency at which the maximum spectrum intensity was obtained was $71 \mathrm{kHz}$. The difference in the calculation was about $4 \%$.

$$
C=\frac{1}{(2 \pi f)^{2} \cdot L}
$$

where $C$ is the capacitance of the capacitor attached to the EM coil, $\mathrm{L}$ is inductance of EM coil, and $\mathrm{f}$ is Drive frequency.

\subsection{Determination of EM Coil Specifications}

The optimum specifications of the EM-coil were experimentally confirmed for the number of permanent magnets of 8 and the driving frequency of $71 \mathrm{kHz}$. The number of turns of the EM-coil was 8 for the transmitter and 15 for the receiver. The diameter of the wire used for making the EM-coil was $0.25 \mathrm{~mm}$ for the transmitter EM-coil and $0.32 \mathrm{~mm}$ for the receiver EM-coil.

\subsection{Optimization of Distance between the Transmitting and Receiving EM Coils}

Figure 5 is an enlarged view of the two permanent magnets of the trial EMAT installed in the circumferential direction of the pipe. The transmitter and receiver EM-coils should overlap in the vertical direction at the same position.

However, the EMAT utilizes the electromagnetic induction phenomenon due to the interaction between the ultrasonic vibration and the bias magnetic field. That is, the EM-coil for the receiver acts an antenna. On the other hand, since a high frequency current is applied to the transmitter EM-coil, the induced electromagnetic waves are much stronger than the induced electromagnetic waves generated by the interaction between the ultrasonic vibration and the biased 
magnetic field. The induced electromagnetic waves due to high frequency electric current are easily detected for the high performance receiver EM-coil. That is, when the transmitter/receiver EM-coil is overlapped in the vertical direction, the influence of the induced electromagnetic wave generated from the transmitter EM-coil is too strong. This means that it is impossible to discriminate the induced electromagnetic wave by the ultrasonic wave vibration. Therefore, as shown in Figure 5, the transmitter and receiver EM-coils were installed side by side in the pipe axis direction, and the relationship between the distance between both EM coils and the received signal intensity was evaluated. Figure 6 shows the experimental result of the spectrum intensity based on the distance between both EM-coils. It shows that the spectrum intensity became a maximum when the distance was $1 \mathrm{~mm}$.
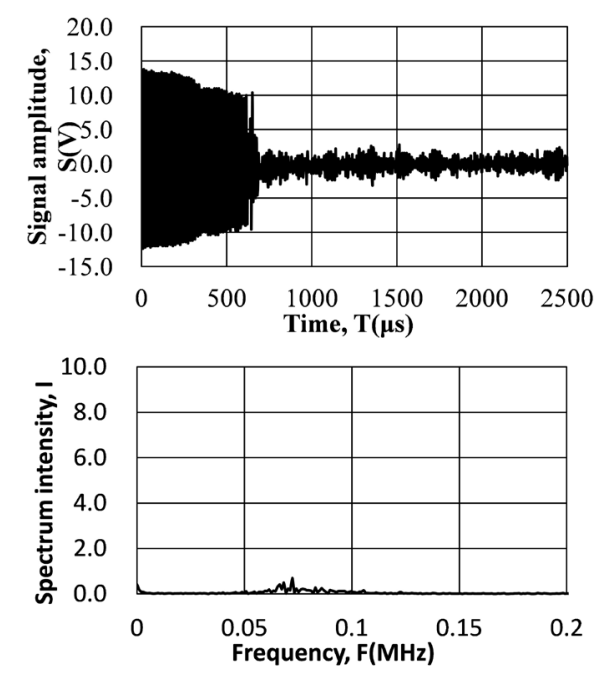

(a)

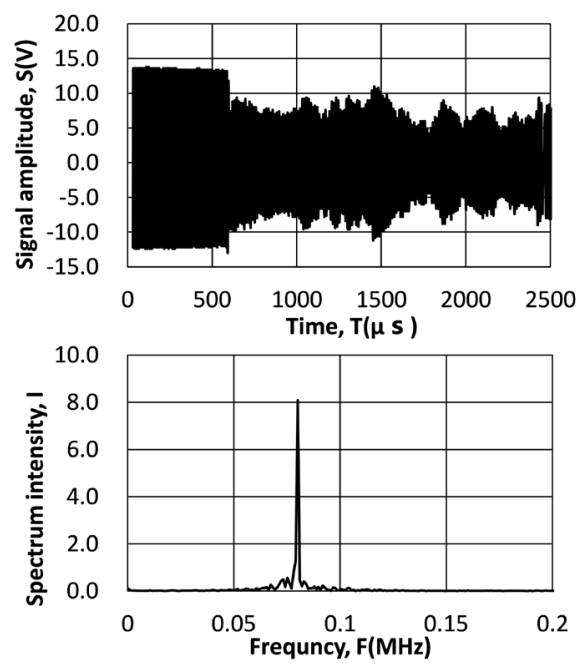

(b)

Figure 3. Example of the received signal (The number of magnets is 8). (a) Non-resonance frequency (59 kHz); (b) Resonance frequency $(71 \mathrm{kHz})$. 


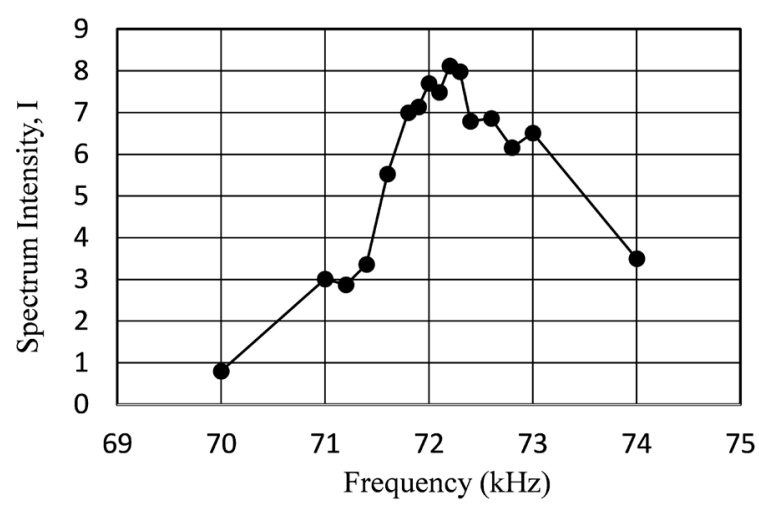

Figure 4. Intensity of the spectrum and the drive frequency.

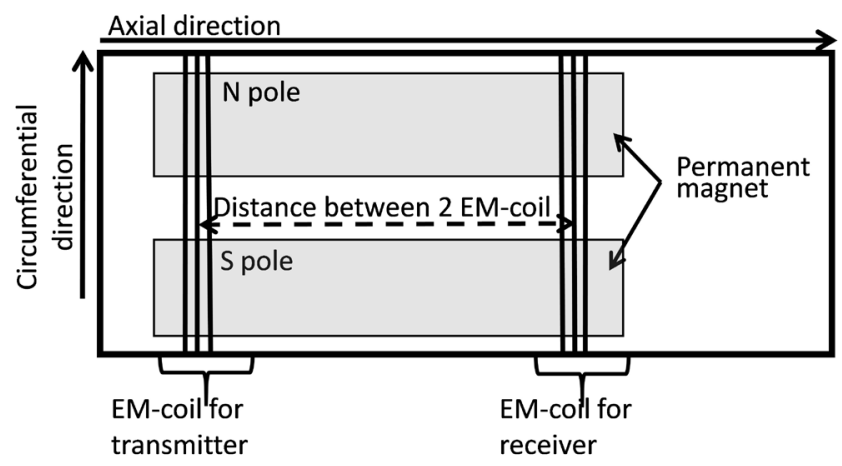

Figure 5. Influence of the electromagnetic wave due to the distance between the transmitter.

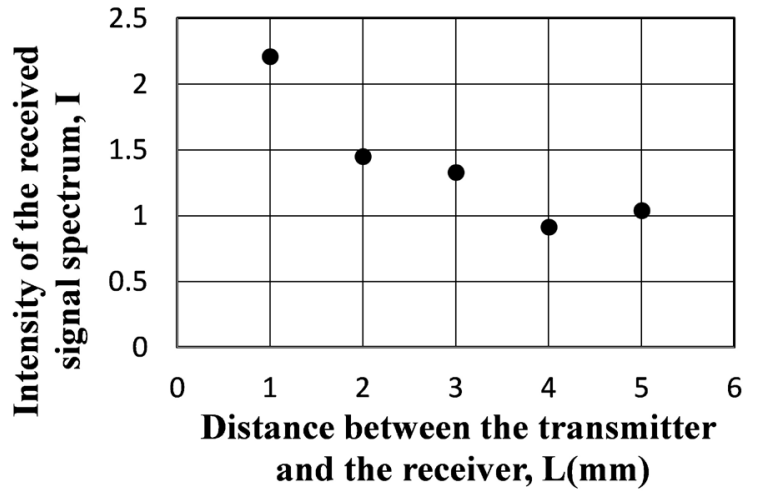

Figure 6. Distance between both EMATs and the signal spectrum intensity.

\section{Wall Thickness Evaluation Test in Stationary State}

As a preliminary experiment for defect detection, an experiment was conducted to confirm the influence of the pipe wall thickness change. Pipes with different wall thicknesses were used. The experiment was also carried out without changing the optimum conditions of the pipe wall thickness of $2 \mathrm{~mm}$ including the driving frequency of $71 \mathrm{kHz}$. Figure 7 shows the relationship between the wall thickness and the spectrum intensity. The spectrum intensity is the average value 
of the measured values at the center of the pipe where the ultrasonic sensor is installed. As a result, the signal intensity due to the change in the wall thickness decreased by about $10 \% / \mathrm{mm}$. Figure 8 shows the relationship between the sensor installation position and the spectrum intensity using a pipe with the wall thickness of $2 \mathrm{~mm}$. It was found that there was a variation in the spectrum intensity of about $20 \%$ depending on the sensor location. This shows that the influence of the pipe position is stronger than that of the pipe thickness.

\section{Measures against Fluctuation of the Received Signal Due to the Sensor Position on the Pipe}

The cause of the signal fluctuation due to the pipe installation position of the sensor is as follows. As shown in Figure 9, a guide wave propagating in the axial direction from the sensor is also generated, then reflected by the pipe edge and returns to the receiver EM-coil. It can happen that both the SH-mode plate wave propagating in the circumferential direction and the axis direction interferes with each other.

Although it is difficult to completely eliminate this effect, as shown in Figure 10(b), a meander line EM-coil was fabricated and all parts of the EM-coil were placed under the permanent magnet. Furthermore, the part of the wire directed in the circumferential direction was not under the permanent magnet in the case of the meander line EM-coil as shown in Figure 10(c).

Figure 11 shows the result of checking the fluctuation of the received signal intensity due to the sensor installation positions of the three kinds of receiver EM-coils shown in Figure 10. The signal intensity according to Figure 10(b) and Figure $10(\mathrm{c})$ has decreased about $40 \%$ because the sensor structure shown in Figure 10(a) can easily detect, in principle, the SH-mode plate wave that propagates in the circumferential direction. However, the intensity fluctuation due to the sensor position in the case of Figure 10(b) and Figure 10(c) decreased compared to the sensor of Figure 10(a). Especially, the reduction in the fluctuation by the EM-coil of Figure 10(c) was remarkable. In other words, the fluctuation rate of the EM-coil of Figure 10(c) decreased about 5\% with respect to the fluctuation rate of the EM-coil of Figure 10(a) of about $21 \%$. Figure 12 shows the relationship between the wall thickness and the received signal intensity when the EM-coil of Figure 10(c) is used and the sensor position is at the pipe center and the pipe end face. The difference between both positions was about $2 \%$. It was confirmed that the influence of the pipe installation position can be reduced.

\section{Pipe Wall Thickness Measurement and the Flaw Detection While Running}

In order to evaluate the influence when the sensor is running, a running experiment was conducted by combining the mechanical scanning system shown in Figure 13. The running speed was $20 \mathrm{~mm} / \mathrm{s}$. The experiments were repeated five 
times under the same conditions using four pipes of different wall thicknesses. The received signal waveform at the specific position in the axial direction was recorded. The results are shown in Figure 14. The evaluation values of the signal intensity, while the sensor system is running on the center part of the pipe are shown. The middle means the center of the pipe and the top means the position $100 \mathrm{~mm}$ from the edge of the pipe in Figure 14. The difference in the evaluation value at the center and end of the pipe was within $5 \%$. Next, it was confirmed that the signal intensity could change where there was a defect in part of the circumferential direction of the pipe. To do this experiment, a drill hole with a different diameter was machined in the pipe thickness direction. The spectrum intensity of the received signal when passing through the drilled hole was evaluated. The results are shown in Figure 15. The measured value might be considered to change due to the relative relationship between the position of the permanent magnet and the position of the drilled hole. Therefore, the pipe was rotated in increments of $30^{\circ}$ in the range of $0^{\circ}$ to $180^{\circ}$ with respect to the reference orientation. The measured values represent the average value and the variation (1 standard deviation) at different positions in the circumferential direction as shown in Figure 15. It is indicated that the system can detect a drill hole of 3 $\mathrm{mm}$ in diameter.

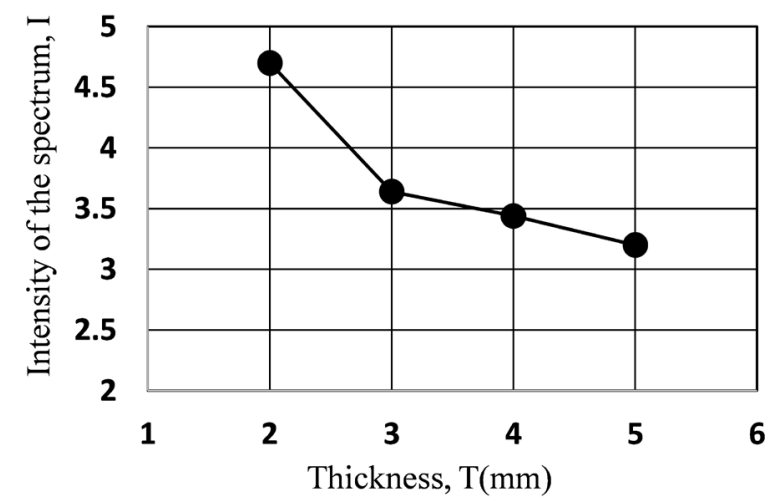

Figure 7. Intensity of the spectrum and the thickness of the pipe.

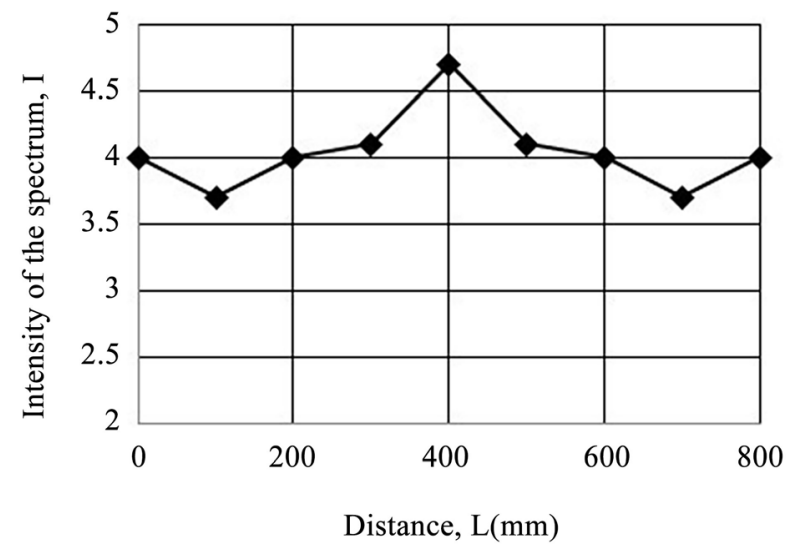

Figure 8. Intensity of spectrum and the positon of the sensor on the pipe. 


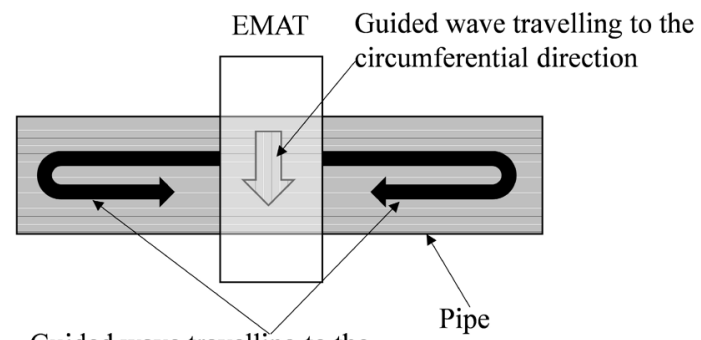

Guided wave travelling to the axis direction

Figure 9. Reason for the signal intensity fluctuation due to the position of the sensor.

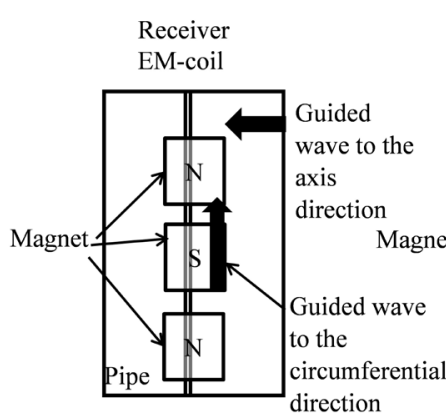

(a)

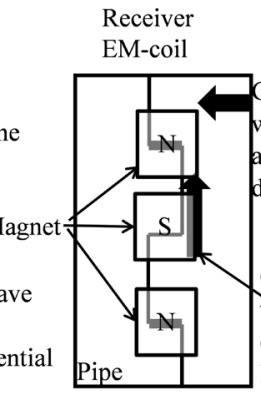

(b)

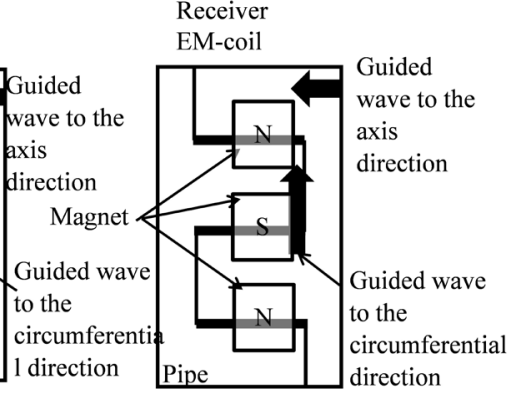

(c)

Figure 10. Improvement of the receiver EM-coil. (a) Circular shape; (b) Comb shape (under the magnet); (c) Comb shape (out of the magnet partially).

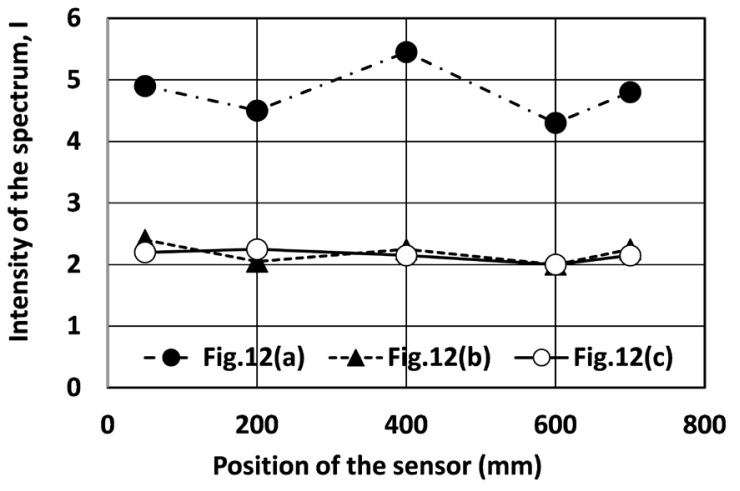

Figure 11. Received signal intensity and the sensor position.

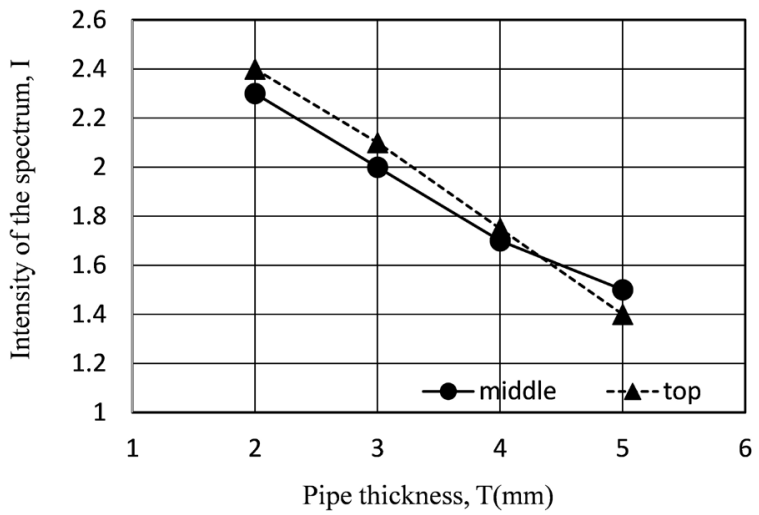

Figure 12. Received signal intensity and the pipe thickness. 


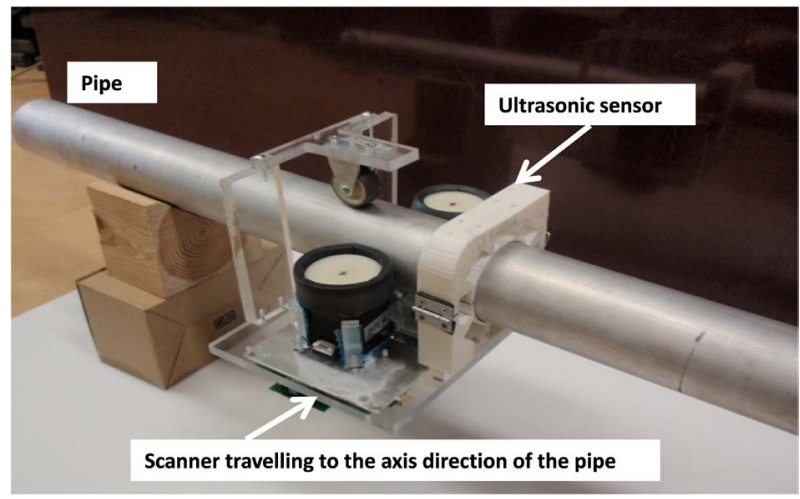

Figure 13. Mechanical scanning system.
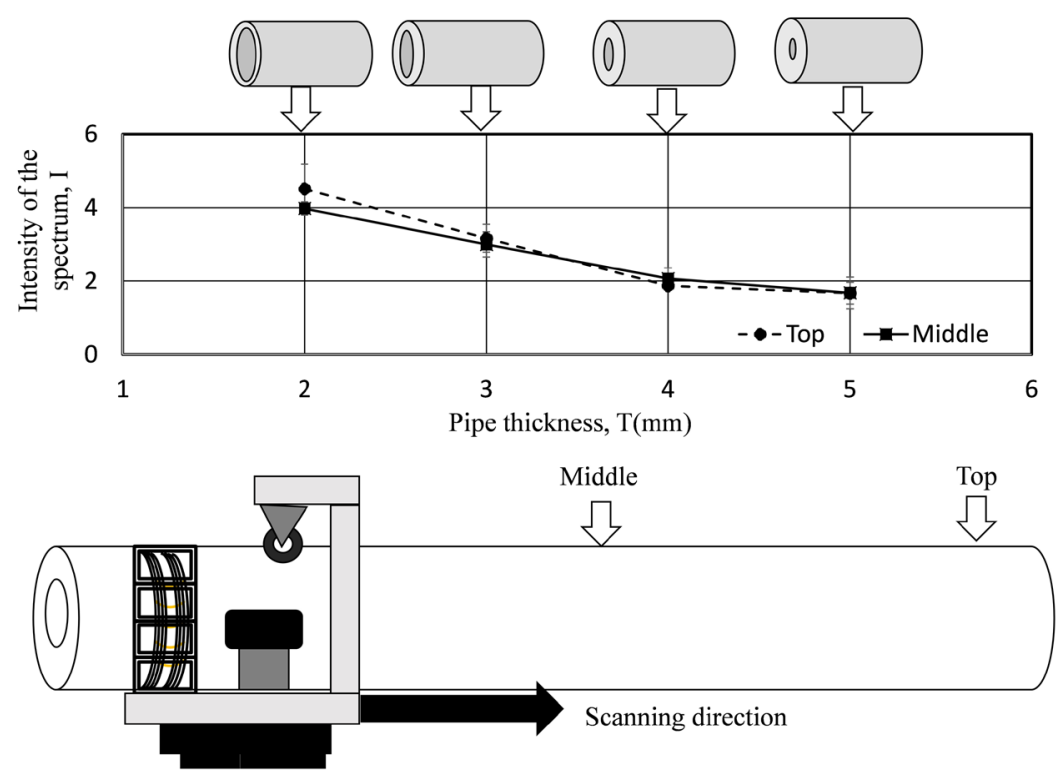

Figure 14. Pipe thickness and signal intensity.

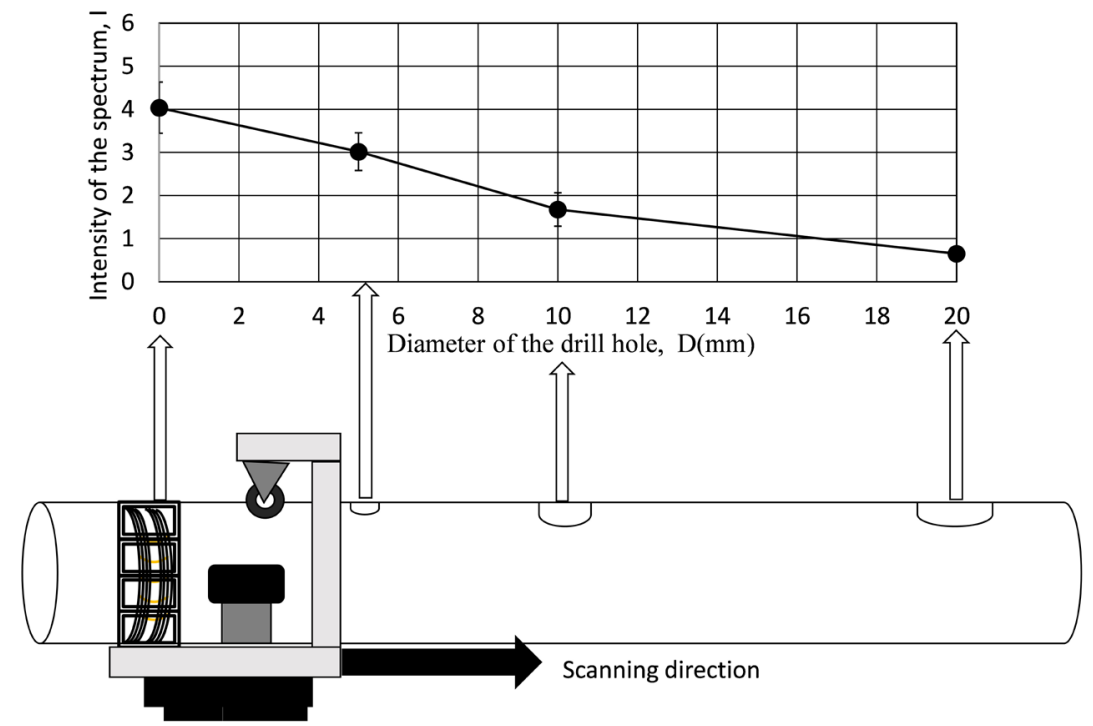

Figure 15. Drill hole diameter and signal intensity. 


\section{Conclusion}

The trial EMAT transmits and receives the SH-mode plate wave propagating in the circumferential direction to detect the presence or absence of defects in the pipe. The trial EMAT consists of an EM-coil wound in the circumferential direction and 8 permanent magnets arranged equidistant in the circumferential direction. In order to make the signal strong, the driving condition was adjusted and synchronized. An aluminum pipe with a diameter of $60 \mathrm{~mm}$ and a wall thickness of $2 \mathrm{~mm}$ was used to determine the drive conditions of the trial EMAT. However, the received signal intensity changed due to the sensor installation position by about $20 \%$. This is because the guided wave propagated not only in the circumferential direction of the pipe but also in the axial direction. As a result of modifying the shape of the receiver EM-coil, the received signal intensity fluctuation could be reduced to about $4 \%$. It was also confirmed that the fluctuation of the received signal intensity is almost the same as the stationary experiment. It was also confirmed that a through drilled hole with a diameter of $3 \mathrm{~mm}$ could be detected even during a running status.

\section{Conflicts of Interest}

The authors declare no conflicts of interest regarding the publication of this paper.

\section{References}

[1] Joseph, L.R. (2002) A Baseline and Vision of Ultrasonic Guided Wave Inspection Potential. Journal of Pressure Vessel Technology, 124, 273-282. http://doi.org/10.1115/1.1491272

[2] Huang, S., Wang, S., Li, W. and Wang, Q. (2016) Electromagnetic Ultrasonic Guided Waves. Springer, Germany. https://doi.org/10.1007/978-981-10-0564-0

[3] Lowe, M.J.S., Alleyne and Cawley, P. (1998) Defect Detection in Pipes Using Guided Waves. Ultrasonics, 36, 147-155. http://doi.org/10.1016/S0041-624X(97)00038-3

[4] Kwun, H. and Teller, C.M. (1994) Detection of Fractured Wires in Steel Cables Using Magnetostrictive Sensors. Material Evaluation, 52, 503-507.

[5] Kwun, H. and Holt, A.E. (1995) Feasibility of Under Lagging Corrosion Detection in Steel Pipe Using the Magnetostrictive Sensor Technique. NDT \& E International, 28, 211-214. https://doi.org/10.1016/0963-8695(95)00019-T

[6] Davies, J., Simonetti, F., Lowe, M. and Cawley, P. (2006) Review of Synthetically Focused Guided Wave Imaging Techniques with Application to Defect Sizing. AIP Conference Proceedings, 820, 142. https://doi.org/10.1063/1.2184522

[7] Wilcox, P.D. (2003) Wilcox: Omni-Directional Guided Wave Transducer Arrays for the Rapid Inspection of Large Areas of Plate Structures. IEEE Transactions on UItrasonics, Ferroelectrics and Frequency Control, 50, 699-709. http://doi.org/10.1109/TUFFC.2003.1209557

[8] Su, Z., Ye, L. and Lu, Y.L. (2006) Guided Lamb Waves for Identification of Damage in Composite Structures. Journal of Sound and Vibration, 295, 753-780. https://doi.org/10.1016/j.jsv.2006.01.020

[9] Hayashi, T., Song, W.-J. and Rose, J.L. (2003) Guided Wave Dispersion Curves for a Bar with an Arbitrary Cross-Section, a Rod and Rail Example. Ultrasonics, 41, 
175-183. https://doi.org/10.1016/S0041-624X(03)00097-0

[10] Liu, G. and Qu, J. (1998) Guided Circumferential Waves in a Circular Annulus. Journal of Applied Mechanics, 65, 424-430. http://doi.org/10.1115/1.2789071

[11] Zhao, X. and Rose, J.L. (2004) Guided Circumferential Shear Horizontal Waves in an Isotropic Hollow Cylinder. The Journal of the Acoustical Society of America, 115, 1912-1916. https://doi.org/10.1121/1.1691037

[12] Valle, C., Qu, J. and Jacob, L.J. (1999) Guided Circumferential Waves in Layered Cylinders. International Journal of Engineering Science, 37, 1369-1387. https://doi.org/10.1016/S0020-7225(98)00133-5

[13] Clough, M., Flemming, M. and Dixon, S. (2017) Circumferential Guided Wave EMAT System for Pipeline Screening Using Shear Horizontal Ultrasound. NDT \& E International, 86, 20-27. https://doi.org/10.1016/j.ndteint.2016.11.010

[14] Nishino, H., Iwata, K. and Ishikawa, M. (2015) A Method of the Wall Thickness Measurement Using Resonant Phenomena of the Circumferential Lamb Waves Generated by Plural Transducers Located Evenly on the Girth. $36^{\text {th }}$ Symposium on Ultrasonic Electrics, Tsukuba, 5-7 November 2015, 3P2-11-1-3P2-11-2.

[15] Hirao, M. and Ogi, H. (2016) Electromagnetic Acoustic Transducers Second Edition. Springer, The Netherlands.

[16] Morita, K. and Nishino, H. (2013) Consideration on the Estimation of the Resonance Frequency of the Circumferential Lamb Waves Generated and Detected by Eight Transducer-Elements Located Evenly on Circumference. The Japan Society of Mechanical Engineering Annual Meeting, Okayama, 9-11 September, J041032-1-J041032-2. 